\title{
Interactive comment on "A combined analysis of geomagnetic data and cosmic ray secondaries in the September 2017 space weather phenomena studies” by Roman Sidorov et al.
}

\section{Anonymous Referee \#2}

Received and published: 20 February 2019

Page and line numbers refer to file angeo-2018-111.pdf, downloaded 21 January 2019.

I apologize to the authors and to the editor for taking so long to prepare these comments.

\section{General comments:}

Summary: I would rate the scientific contribution of the manuscript as "may have potential after additional work and resubmission," because while an interesting way of looking at the data is described, it is far from clear to this reader exactly what we are to learn from this, and how we would tell when to have confidence in any such conclusions. 
This work brings a new sensor, the URAGAN muon hodoscope, to the study of space weather phenomena, and it describes a mathematical technique, the "generalized characteristic function," that can be used to compare time series of observed quantities (including the hodoscope data) that have different dimensions and dynamic ranges. There may indeed be potential to learn new things about space weather by bringing these to bear on it. However, I did not find sufficient description in the paper of how to use the generalized characteristic function to draw specific, quantitative conclusions about the correlations of such time series.

Specific comments:

The paper spends a significant amount of space on discussing how to "standardize" each time series, normalizing the data to bring all time series of interest into a state (dimensionless, and with similar dynamic ranges) that allows more direct comparison. Lines 3-25 on page 5 and lines 5-19 of page 7, and the entirety of figure 3, separate the time series of interest here into those with normal distribution and those with lognormal distribution, presenting a quantitative test (equations 3-4) for how well these functional forms represent each time series.

But what are we to do with these standardized time series once we have generated them? Equation 1 defines a "generalized characteristic function" as a linear combination of standardized time series, but all that is said about the weight coefficients is that they "depend[] on the properties of a particular data set, its physical origin and veracity." In lines 31-32 of page 5 it is said to be "possible to adjust the data set contributions [to the GCF] using weight coefficients with the standardized time series," and a few lines later it is mentioned that these coefficients can be negative as well as positive, but how does one choose their values? The two GCFs defined in equation 4 on page 7 (which should be equation 5) and plotted in panel (e) of figure 1 have weight coefficients of plus or minus unity; why? Line 33 of page 7 through line 2 of page 8 says that "weight coefficients for particular time series ... can be customized in order to reveal specific patterns of their behavior, such as uplifts, mistiming and others," but what such

Interactive

comment 
And once we have these GCFs, what do we learn by comparing them? No quantitative correlation tests between GCFs are presented, and I do not see how putting the slowlyvarying muon trend time series in each GCF tells us anything that we would not see from a simple comparison of the (standardized) TEC and Dst time series. For example, what does the muon data add to the discussion in lines 5-11 of page 8? In panel (e) of figure 1, the "slight uplifts" in GCF G2 and their absence in G1 give us the same information as is seen in comparing panels (c) and (d) directly. In lines 19-20 of page 8 it is said that "the muon flux intensity increase ... can be related to the change of conditions in the ionosphere"; how do we obtain this relation from the GCFs? In lines 20-21 of page 7 it is stated that the two GCFS are "used in order to estimate the correlation between" muons and Dst and between muons and TEC. Where is this estimate presented in the paper? I do not have access to the Troyan \& Kiselev book so that I can go look up the GCF technique; lines 26-28 of page 4 say it is "widely implemented in exploration geophysics," but a space weather audience for the paper will need more detailed and quantitative explanations than are provided.

Finally, if questions of timing are to be addressed, the details of how the muon "trend ... was built using a piecewise-linear approximation" need to be given, in order to ensure that any features in the muon trend data whose timing is compared with features in other data sets are not simply artifacts of the way the approximation was constructed.

Technical corrections:

Generally the paper is clear, though additional proofreading after revision would be helpful. Here are a few minor suggestions I noted in passing.

On line 13 of page 1, "the ones of the most powerful ... events" has a few extra words; perhaps "some of the most powerful ... events."

On line 2 of page 5, "veracity" is not clear - reliability? Accuracy?
ANGEOD

Interactive

comment
Printer-friendly version

Discussion paper 
On page 7, equation 4 should be numbered equation 5.

Reference \#10 still has http://dx.doi.org/ attached to its DOI number.

ANGEOD

Interactive comment on Ann. Geophys. Discuss., https://doi.org/10.5194/angeo-2018-111, 2018.

Interactive

comment 